\title{
On representations of a number as a sum of three triangles
}

by

Michael D. Hirschiorn (Sydney, N.S.W.) and James A. Sellers (Cedarville, Ohio)

1. Introduction. Let $t(n)$ be the number of representations of $n$ as the sum of three triangular numbers. Then

$$
\sum_{n \geq 0} t(n) q^{n}=\left(\frac{\left(q^{2} ; q^{2}\right)_{\infty}}{\left(q ; q^{2}\right)_{\infty}}\right)^{3} .
$$

Gauss [2] showed that $t(n)>0$, in other words, every number is the sum of three triangles. Recently, George E. Andrews [1] provided a proof of this fact via $q$-series.

We shall show that $t(n)$ satisfies infinitely many arithmetic identities. Thus, for $\lambda \geq 1$,

$$
\begin{aligned}
t\left(3^{2 \lambda+1} n+\left(11 \cdot 3^{2 \lambda}-3\right) / 8\right) & =3^{\lambda} \cdot t(3 n+1), \\
t\left(3^{2 \lambda+1} n+\left(19 \cdot 3^{2 \lambda}-3\right) / 8\right) & =\left(2 \cdot 3^{\lambda}-1\right) t(3 n+2), \\
t\left(3^{2 \lambda+2} n+\left(3^{2 \lambda+1}-3\right) / 8\right) & =\left(\left(3^{\lambda+1}-1\right) / 2\right) t(9 n)
\end{aligned}
$$

and

$$
t\left(3^{2 \lambda+2} n+\left(17 \cdot 3^{2 \lambda+1}-3\right) / 8\right)=\left(\left(3^{\lambda+1}-1\right) / 2\right) t(9 n+6) .
$$

It should be noted that $t(n)=r(8 n+3)$, where $r(n)$ is the number of representations of $n$ in the form $n=k^{2}+l^{2}+m^{2}$ with $k, l, m$ odd and positive. Indeed,

$$
n=\left(r^{2}+r\right) / 2+\left(s^{2}+s\right) / 2+\left(t^{2}+t\right) / 2
$$

is equivalent to

$$
8 n+3=(2 r+1)^{2}+(2 s+1)^{2}+(2 t+1)^{2} .
$$

This research was begun while the first author was enjoying leave. 
Our results can be written in terms of $r(n)$. Thus, we have

$$
r\left(9^{\lambda} n\right)= \begin{cases}3^{\lambda} \cdot r(n) & \text { if } n \equiv 11(\bmod 24), \\ \left(2 \cdot 3^{\lambda}-1\right) r(n) & \text { if } n \equiv 19(\bmod 24), \\ \left(\left(3^{\lambda+1}-1\right) / 2\right) r(n) & \text { if } n \equiv 3 \text { or } 51(\bmod 72) .\end{cases}
$$

We also obtain the generating function formulae

$$
\begin{gathered}
\sum_{n \geq 0} t(3 n+1) q^{n}=3 \frac{\left(q^{3} ; q^{3}\right)_{\infty}^{3}}{\left(q ; q^{2}\right)_{\infty}^{2}}, \quad \sum_{n \geq 0} t(3 n+2) q^{n}=3 \frac{\left(q^{6} ; q^{6}\right)_{\infty}^{3}}{\left(q ; q^{2}\right)_{\infty}} \\
\sum_{n \geq 0} t(9 n) q^{n}=\frac{\left(q^{3} ; q^{3}\right)_{\infty}}{\left(q ; q^{6}\right)_{\infty}\left(q^{5} ; q^{6}\right)_{\infty}}\left\{1+6 \sum_{n \geq 1}\left(\frac{q^{3 n-2}}{1-q^{3 n-2}}-\frac{q^{3 n-1}}{1-q^{3 n-1}}\right)\right\},
\end{gathered}
$$

and

$$
\sum_{n \geq 0} t(9 n+6) q^{n}=6 \frac{\left(q^{3} ; q^{6}\right)_{\infty}^{2}\left(q^{6} ; q^{6}\right)_{\infty}^{4}}{(q ; q)_{\infty}}
$$

2. The generating functions for $t(3 n+1)$ and $t(3 n+2)$. We have $t(3 n+1)=r(24 n+11)$, so we need to consider

$$
24 n+11=k^{2}+l^{2}+m^{2}
$$

with $k, l, m$ odd and positive. Modulo 6 , this becomes

$$
k^{2}+l^{2}+m^{2} \equiv 5(\bmod 6) .
$$

This has the solutions, together with permutations,

$$
(k, l, m) \equiv( \pm 1, \pm 1,3)(\bmod 6) .
$$

Conversely, if $(k, l, m) \equiv( \pm 1, \pm 1,3)(\bmod 6)$ then $k^{2}+l^{2}+m^{2} \equiv 5(\bmod 24)$.

It follows that

$$
\begin{aligned}
\sum_{n \geq 0} t(3 n+1) q^{24 n+11} & \\
= & \sum_{n \geq 0} r(24 n+11) q^{24 n+11}=3 \sum_{m \geq 0} q^{(6 k+1)^{2}+(6 l+1)^{2}+(6 m+3)^{2}} .
\end{aligned}
$$

Here we have used the facts that

$$
\sum_{\substack{k>0 \\ k \equiv \pm 1(\bmod 6)}} q^{k^{2}}=\sum q^{(6 k+1)^{2}} \text { and } \sum_{\substack{k>0 \\ k \equiv 3(\bmod 6)}} q^{k^{2}}=\sum_{k \geq 0} q^{(6 k+3)^{2}} .
$$

Thus we have

$$
\sum_{n \geq 0} t(3 n+1) q^{24 n+11}=3 q^{11} \sum_{m \geq 0} q^{36 k^{2}+12 k+36 l^{2}+12 l+36 m^{2}+36 m}
$$


SO

$$
\begin{aligned}
\sum_{n \geq 0} t(3 n+1) q^{n} & =3 \sum_{m \geq 0} q^{\left(3 k^{2}+k\right) / 2+\left(3 l^{2}+l\right) / 2+3\left(\left(m^{2}+m\right) / 2\right)} \\
& =3\left(-q ; q^{2}\right)_{\infty}^{2}\left(-q^{2} ; q^{3}\right)_{\infty}^{2}\left(q^{3} ; q^{3}\right)_{\infty}^{2} \frac{\left(q^{6} ; q^{6}\right)_{\infty}}{\left(q^{3} ; q^{6}\right)_{\infty}} \\
& =3 \frac{\left(q^{3} ; q^{3}\right)_{\infty}^{3}}{\left(q ; q^{2}\right)_{\infty}^{2}}
\end{aligned}
$$

Similarly we find that

$$
\begin{aligned}
\sum_{n \geq 0} t(3 n+2) q^{24 n+19} & =\sum_{n \geq 0} r(24 n+19) q^{24 n+19} \\
& =3 \sum_{l, m \geq 0} q^{(6 k+1)^{2}+(6 l+3)^{2}+(6 m+3)^{2}} \\
& =3 q^{19} \sum_{l, m \geq 0} q^{36 k^{2}+12 k+36 l^{2}+36 l+36 m^{2}+36 m}
\end{aligned}
$$

SO

$$
\begin{aligned}
\sum_{n \geq 0} t(3 n+2) q^{n} & =3 \sum_{l, m \geq 0} q^{\left(3 k^{2}+k\right) / 2+3\left(\left(l^{2}+l\right) / 2\right)+3\left(\left(m^{2}+m\right) / 2\right)} \\
& =3\left(-q ; q^{3}\right)_{\infty}\left(-q^{2} ; q^{3}\right)_{\infty}\left(q^{3} ; q^{3}\right)_{\infty}\left(\frac{\left(q^{6} ; q^{6}\right)_{\infty}}{\left(q^{3} ; q^{6}\right)_{\infty}}\right)^{2} \\
& =3 \frac{\left(q^{6} ; q^{6}\right)_{\infty}^{3}}{\left(q ; q^{2}\right)_{\infty}}
\end{aligned}
$$

3. The generating functions for $t(9 n)$ and $t(9 n+6)$. We have $t(9 n)=$ $r(72 n+3)$, so we need to consider

$$
72 n+3=k^{2}+l^{2}+m^{2}
$$

with $k, l, m$ odd and positive. Modulo 18, this becomes

$$
k^{2}+l^{2}+m^{2} \equiv 3(\bmod 18)
$$

The only solutions of this are, together with permutations,

$(k, l, m)$

$$
\equiv( \pm 1, \pm 1, \pm 1),( \pm 5, \pm 5, \pm 5),( \pm 7, \pm 7, \pm 7) \text { or }( \pm 1, \pm 5, \pm 7)(\bmod 18)
$$

Conversely, if

$(k, l, m)$

$$
\equiv( \pm 1, \pm 1, \pm 1),( \pm 5, \pm 5, \pm 5),( \pm 7, \pm 7, \pm 7) \text { or }( \pm 1, \pm 5, \pm 7)(\bmod 18)
$$

then $k^{2}+l^{2}+m^{2} \equiv 3(\bmod 72)$. Thus, 


$$
\begin{aligned}
\sum_{n \geq 0} t(9 n) q^{72 n+3}= & \sum_{n \geq 0} r(72 n+3) q^{72 n+3} \\
= & \sum q^{(18 k+1)^{2}+(18 l+1)^{2}+(18 m+1)^{2}} \\
& +\sum q^{(18 k-5)^{2}+(18 l-5)^{2}+(18 m-5)^{2}} \\
& +\sum q^{(18 k+7)^{2}+(18 l+7)^{2}+(18 m+7)^{2}} \\
& +6 \sum q^{(18 k+1)^{2}+(18 l-5)^{2}+(18 m+7)^{2}} \\
= & \sum_{k+l+m \equiv 0(\bmod 3)} q^{(6 k+1)^{2}+(6 l+1)^{2}+(6 m+1)^{2}}
\end{aligned}
$$

If we set $k+l+m=3 u, 2 k-l-m=3 v, l-m=w$ then $v \equiv w(\bmod 2)$,

$$
\begin{aligned}
& (6 k+1)^{2}+(6 l+1)^{2}+(6 m+1)^{2} \\
& \quad=12(k+l+m)^{2}+6(2 k-l-m)^{2}+18(l-m)^{2}+12(k+l+m)+3 \\
& =12(3 u)^{2}+6(3 v)^{2}+18 w^{2}+12(3 u)+3 \\
& \quad=108 u^{2}+54 v^{2}+18 w^{2}+36 u+3
\end{aligned}
$$

and

That is,

$$
\sum_{n \geq 0} t(9 n) q^{72 n+3}=\sum_{v \equiv w(\bmod 2)} q^{108 u^{2}+54 v^{2}+18 w^{2}+36 u+3} .
$$

$$
\begin{array}{rl}
\sum_{n \geq 0} & t(9 n) q^{n} \\
& =\sum q^{\left(3 u^{2}+u\right) / 2} \sum_{v \equiv w(\bmod 2)} q^{\left(3 v^{2}+w^{2}\right) / 4} \\
& =\sum q^{\left(3 u^{2}+u\right) / 2} \sum q^{\left(3(s+t)^{2}+(s-t)^{2}\right) / 4}=\sum q^{\left(3 u^{2}+u\right) / 2} \sum q^{s^{2}+s t+t^{2}} \\
& =\left(-q ; q^{3}\right)_{\infty}\left(-q^{2} ; q^{3}\right)_{\infty}\left(q^{3} ; q^{3}\right)_{\infty}\left\{1+6 \sum_{n \geq 1}\left(\frac{q^{3 n-2}}{1-q^{3 n-2}}-\frac{q^{3 n-1}}{1-q^{3 n-1}}\right)\right\} \\
& =\frac{\left(q^{3} ; q^{3}\right)_{\infty}}{\left(q ; q^{6}\right)_{\infty}\left(q^{5} ; q^{6}\right)_{\infty}}\left\{1+6 \sum_{n \geq 1}\left(\frac{q^{3 n-2}}{1-q^{3 n-2}}-\frac{q^{3 n-1}}{1-q^{3 n-1}}\right)\right\} .
\end{array}
$$

(For a proof that

$$
\sum q^{s^{2}+s t+t^{2}}=1+6 \sum_{n \geq 1}\left(\frac{q^{3 n-2}}{1-q^{3 n-2}}-\frac{q^{3 n-1}}{1-q^{3 n-1}}\right)
$$

see the Appendix.) 
In similar fashion,

$$
\begin{aligned}
\sum_{n \geq 0} t(9 n+6) q^{72 n+51}= & \sum_{n \geq 0} r(72 n+51) q^{72 n+51} \\
= & 3 \sum q^{(18 k+1)^{2}+(18 l-5)^{2}+(18 m-5)^{2}} \\
& +3 \sum q^{(18 k-5)^{2}+(18 l+7)^{2}+(18 m+7)^{2}} \\
& +3 \sum q^{(18 k+7)^{2}+(18 l+1)^{2}+(18 m+1)^{2}} \\
= & \sum_{k+l+m \equiv 1(\bmod 3)} q^{(6 k+1)^{2}+(6 l+1)^{2}+(6 m+1)^{2}} .
\end{aligned}
$$

If we set $k+l+m=3 u+1,2 k-l-m=3 v-1, l-m=w$ then $v \not \equiv w$ $(\bmod 2)$,

$$
\begin{aligned}
& (6 k+1)^{2}+(6 l+1)^{2}+(6 m+1)^{2} \\
& \quad=12(k+l+m)^{2}+6(2 k-l-m)^{2}+18(l-m)^{2}+12(k+l+m)+3 \\
& \quad=12(3 u+1)^{2}+6(3 v-1)^{2}+18 w^{2}+12(3 u+1)+3 \\
& \quad=108 u^{2}+54 v^{2}+18 w^{2}+108 u-36 v+33
\end{aligned}
$$

and

$$
\sum_{n \geq 0} t(9 n+6) q^{72 n+51}=\sum_{v \neq w(\bmod 2)} q^{108 u^{2}+54 v^{2}+18 w^{2}+108 u-36 v+33} .
$$

That is,

$$
\begin{aligned}
\sum_{n \geq 0} t(9 n+6) q^{n} & =\sum q^{3\left(\left(u^{2}+u\right) / 2\right)} \sum_{v \neq w(\bmod 2)} q^{\left(3 v^{2}+w^{2}-2 v-1\right) / 4} \\
& =\sum q^{3\left(\left(u^{2}+u\right) / 2\right)} \sum q^{\left(3(s+t+1)^{2}+(s-t)^{2}-2(s+t+1)-1\right) / 4} \\
& =\sum q^{3\left(\left(u^{2}+u\right) / 2\right)} \sum q^{s^{2}+s t+t^{2}+s+t} \\
& =2 \frac{\left(q^{6} ; q^{6}\right)_{\infty}}{\left(q^{3} ; q^{6}\right)_{\infty}} \cdot 3 \frac{\left(q^{3} ; q^{3}\right)_{\infty}^{3}}{(q ; q)_{\infty}}=6 \frac{\left(q^{3} ; q^{6}\right)_{\infty}^{2}\left(q^{6} ; q^{6}\right)_{\infty}^{4}}{(q ; q)_{\infty}} .
\end{aligned}
$$

(For a proof that

see the Appendix.)

$$
\sum q^{s^{2}+s t+t^{2}+s+t}=3 \frac{\left(q^{3} ; q^{3}\right)_{\infty}^{3}}{(q ; q)_{\infty}}
$$

4. Proof of the main result in the case $\lambda=1$. We want to show that

$$
\begin{aligned}
& t(27 n+12)=3 t(3 n+1), \\
& t(27 n+21)=5 t(3 n+2),
\end{aligned}
$$




$$
\begin{aligned}
t(81 n+3) & =4 t(9 n), \\
t(81 n+57) & =4 t(9 n+6) .
\end{aligned}
$$

As we shall see, it is crucial for us to prove

$$
\sum_{n \geq 0} t(27 n+3) q^{n}=4 \sum_{n \geq 0} t(3 n) q^{n}-3 \sum_{n \geq 0} t(n) q^{3 n+1} .
$$

The third and fourth of the above relations follow from $(*)$ on comparing coefficients of $q^{3 n}$ and $q^{3 n+2}$ respectively. Also, we shall require $(*)$ in Section 5 to prove the main result for $\lambda>1$.

We shall prove $(*)$ in full detail, and outline the proofs of the first and second relations above.

First,

$$
\begin{aligned}
\sum_{n \geq 0} t(3 n) q^{24 n+3}= & \sum_{n \geq 0} r(24 n+3) q^{24 n+3} \\
= & \sum q^{(6 k+1)^{2}+(6 l+1)^{2}+(6 m+1)^{2}} \\
& +\sum_{k, l, m \geq 0} q^{(6 k+3)^{2}+(6 l+3)^{2}+(6 m+3)^{2}} \\
= & q^{3} \sum^{36 k^{2}+12 k+36 l^{2}+12 l+36 m^{2}+12 m} \\
& +q^{27} \sum_{k, l, m \geq 0} q^{36 k^{2}+36 k+36 l^{2}+36 l+36 m^{2}+36 m}
\end{aligned}
$$

so

$$
\begin{aligned}
\sum_{n \geq 0} t(3 n) q^{n}= & \sum q^{\left(3 k^{2}+k\right) / 2+\left(3 l^{2}+l\right) / 2+\left(3 m^{2}+m\right) / 2} \\
& +q \sum_{k, l, m \geq 0} q^{3\left(\left(k^{2}+k\right) / 2\right)+3\left(\left(l^{2}+l\right) / 2\right)+3\left(\left(m^{2}+m\right) / 2\right)} \\
= & \sum q^{\left(3 k^{2}+k\right) / 2+\left(3 l^{2}+l\right) / 2+\left(3 m^{2}+m\right) / 2} \\
& +\sum_{n \geq 0} t(n) q^{3 n+1} .
\end{aligned}
$$

Next, $t(27 n+3)=r(216 n+27)$, so we need to consider

$$
216 n+27=k^{2}+l^{2}+m^{2}
$$

with $k, l, m$ odd and positive. Modulo 54 , this becomes

$$
k^{2}+l^{2}+m^{2} \equiv 27(\bmod 54)
$$

and we find that

$$
\sum_{n \geq 0} t(27 n+3) q^{216 n+27}=\sum_{n \geq 0} r(216 n+27) q^{216 n+27}
$$




$$
\begin{aligned}
=3\{ & \sum q^{(54 k-23)^{2}+(54 l-17)^{2}+(54 m-17)^{2}}+\sum q^{(54 k-17)^{2}+(54 l+25)^{2}+(54 m+25)^{2}} \\
& +\sum q^{(54 k-11)^{2}+(54 l+13)^{2}+(54 m+13)^{2}}+\sum q^{(54 k-5)^{2}+(54 l+1)^{2}+(54 m+1)^{2}} \\
& +\sum q^{(54 k+1)^{2}+(54 l-11)^{2}+(54 m-11)^{2}}+\sum q^{(54 k+7)^{2}+(54 l-23)^{2}+(54 m-23)^{2}} \\
& +\sum q^{(54 k+13)^{2}+(54 l+19)^{2}+(54 m+19)^{2}}+\sum q^{(54 k+19)^{2}+(54 l+7)^{2}+(54 m+7)^{2}} \\
& \left.+\sum q^{(54 k+25)^{2}+(54 l-5)^{2}+(54 m-5)^{2}}\right\} \\
+6\{ & \sum q^{(54 k-23)^{2}+(54 l+19)^{2}+(54 m+1)^{2}}+\sum q^{(54 k-17)^{2}+(54 l+7)^{2}+(54 m-11)^{2}} \\
& +\sum q^{(54 k-11)^{2}+(54 l-5)^{2}+(54 m-23)^{2}}+\sum q^{(54 k-5)^{2}+(54 l-17)^{2}+(54 m+19)^{2}} \\
& +\sum q^{(54 k+1)^{2}+(54 l+25)^{2}+(54 m+7)^{2}}+\sum q^{(54 k+7)^{2}+(54 l+13)^{2}+(54 m-5)^{2}} \\
& +\sum q^{(54 k+13)^{2}+(54 l+1)^{2}+(54 m-17)^{2}}+\sum q^{(54 k+19)^{2}+(54 l-11)^{2}+(54 m+25)^{2}} \\
& \left.+\sum q^{(54 k+25)^{2}+(54 l-23)^{2}+(54 m+13)^{2}}\right\} \\
& +\sum q^{(18 k+3)^{2}+(18 l+3)^{2}+(18 m+3)^{2}} \\
& +\sum q_{k, l, m \geq 0} q^{(18 k+9)^{2}+(18 l+9)^{2}+(18 m+9)^{2}} .
\end{aligned}
$$

The last two terms constitute

$$
\sum_{n \geq 0} t(3 n) q^{216 n+27}
$$

so we have

$$
\begin{aligned}
\sum_{n \geq 0} t(27 n+3) q^{216 n+27} & -\sum_{n \geq 0} t(3 n) q^{216 n+27} \\
= & 3 \sum_{\substack{4 k+l+m \equiv-4(\bmod 9) \\
l-m \equiv 0(\bmod 3)}} q^{(6 k+1)^{2}+(6 l+1)^{2}+(6 m+1)^{2}} .
\end{aligned}
$$

If we set $4 k+l+m=9 u-4,-k+2 l+2 m=9 v+1, l-m=3 w$ then $u \equiv w(\bmod 2)$,

$$
\begin{aligned}
(6 k+1)^{2}+ & (6 l+1)^{2}+(6 m+1)^{2} \\
= & 2(4 k+l+m)^{2}+4(-k+2 l+2 m)^{2}+18(l-m)^{2} \\
& +4(4 k+l+m)+4(-k+2 l+2 m)+3
\end{aligned}
$$




$$
\begin{aligned}
& =2(9 u-4)^{2}+4(9 v+1)^{2}+18(3 w)^{2}+4(9 u-4)+4(9 v+1)+3 \\
& =162 u^{2}+324 v^{2}+162 w^{2}-108 u+108 v+27
\end{aligned}
$$

and

$$
\begin{aligned}
\sum_{n \geq 0} t(27 n+3) q^{216 n+27} & -\sum_{n \geq 0} t(3 n) q^{216 n+27} \\
& =3 \sum_{u \equiv w(\bmod 2)} q^{162 u^{2}+324 v^{2}+162 w^{2}-108 u+108 v+27} .
\end{aligned}
$$

That is,

$$
\begin{array}{rl}
\sum_{n \geq 0} t(27 n+3) q^{n}-\sum_{n \geq 0} & t(3 n) q^{n} \\
& =3 \sum q^{\left(3 v^{2}+v\right) / 2} \sum_{u \equiv w(\bmod 2} q^{\left(3 u^{2}+3 w^{2}-2 u\right) / 4} \\
& =3 \sum q^{\left(3 v^{2}+v\right) / 2} \sum q^{\left(3(s+t)^{2}+3(s-t)^{2}-2(s+t)\right) / 4} \\
& =3 \sum q^{\left(3 v^{2}+v\right) / 2+\left(3 s^{2}-s\right) / 2+\left(3 t^{2}-t\right) / 2} \\
& =3\left(\sum_{n \geq 0} t(3 n) q^{n}-\sum_{n \geq 0} t(n) q^{3 n+1}\right),
\end{array}
$$

so

$$
\sum_{n \geq 0} t(27 n+3) q^{n}=4 \sum_{n \geq 0} t(3 n) q^{n}-3 \sum_{n \geq 0} t(n) q^{3 n+1} .
$$

In the same way, we can show

$$
\begin{aligned}
\sum_{n \geq 0} t(27 n+12) q^{216 n+99}-\sum_{n \geq 0} t(3 n+1) q^{216 n+99} & =3 \sum_{\substack{4 k+l+m \equiv 2(\bmod 9) \\
l-m \equiv 0(\bmod 3)}} q^{(6 k+1)^{2}+(6 l+1)^{2}+(6 m+1)^{2}}
\end{aligned}
$$

from which it follows that

$$
\begin{aligned}
\sum_{n \geq 0} t(27 n+12) q^{n}- & \sum_{n \geq 0} t(3 n+1) q^{n} \\
& =3 \sum q^{3\left(\left(v^{2}+v\right) / 2\right)} \sum_{u \equiv w(\bmod 2)} q^{\left(3 u^{2}+3 w^{2}+2 u\right) / 4} \\
& =6 \sum_{v \geq 0} q^{3\left(\left(v^{2}+v\right) / 2\right)+\left(3 s^{2}+s\right) / 2+\left(3 t^{2}+t\right) / 2} \\
& =2 \sum_{n \geq 0} t(3 n+1) q^{n}
\end{aligned}
$$


and

$$
\begin{aligned}
\sum_{n \geq 0} t(27 n+21) q^{216 n+171}- & \sum_{n \geq 0} t(3 n+2) q^{216 n+171} \\
=3 & \sum_{\substack{4 k+l+m \equiv-1(\bmod 9) \\
l-m \equiv 0(\bmod 3)}} q^{(6 k+1)^{2}+(6 l+1)^{2}+(6 m+1)^{2}}
\end{aligned}
$$

from which it follows that

$$
\begin{array}{rl}
\sum_{n \geq 0} t(27 n+21) q^{n}-\sum_{n \geq 0} & t(3 n+2) q^{n} \\
= & 3 \sum q^{\left(3 v^{2}+v\right) / 2} \sum_{u \neq w(\bmod 2)} q^{\left(3 u^{2}+3 w^{2}-3\right) / 4} \\
& =3 \sum q^{\left(3 v^{2}+v\right) / 2+3\left(\left(s^{2}+s\right) / 2\right)+3\left(\left(t^{2}+t\right) / 2\right)} \\
& =12 \sum_{s, t \geq 0} q^{\left(3 v^{2}+v\right) / 2+3\left(\left(s^{2}+s\right) / 2\right)+3\left(\left(t^{2}+t\right) / 2\right)} \\
& =4 \sum_{n \geq 0} t(3 n+2) q^{n} .
\end{array}
$$

Thus the first and second relations hold.

5. Proof of the main result for $\lambda>1$. We have shown that

$$
r\left(9^{\lambda} n\right)= \begin{cases}3^{\lambda} r(n) & \text { if } n \equiv 11(\bmod 24), \\ \left(2 \cdot 3^{\lambda}-1\right) r(n) & \text { if } n \equiv 19(\bmod 24), \\ \left(\left(3^{\lambda+1}-1\right) / 2\right) r(n) & \text { if } n \equiv 3 \text { or } 51(\bmod 72)\end{cases}
$$

for $\lambda=1$, and it is trivially true for $\lambda=0$.

Suppose now that $\lambda \geq 2$, and that the result is true for $\lambda-1$ and $\lambda-2$.

We have

$$
\sum_{n \geq 0} t(27 n+3) q^{n}=4 \sum_{n \geq 0} t(3 n) q^{n}-3 \sum_{n \geq 0} t(n) q^{3 n+1} .
$$

If we consider the coefficient of $q^{3 n+1}$ we obtain

$$
t(81 n+30)=4 t(9 n+3)-3 t(n)
$$

or

$$
r(648 n+243)=4 r(72 n+27)-3 r(8 n+3),
$$

or

$$
r(81(8 n+3))=4 r(9(8 n+3))-3 r(8 n+3) .
$$

It follows that for all $n$,

$$
r(81 n)=4 r(9 n)-3 r(n),
$$


for if $n \not \equiv 3(\bmod 8)$ then $81 n \equiv 9 n \equiv n \not \equiv 3(\bmod 8)$ so $r(81 n)=r(9 n)=$ $r(n)=0$ and the result is trivially true.

It follows that for every $\lambda \geq 2$,

$$
r\left(9^{\lambda} n\right)=4 r\left(9^{\lambda-1} n\right)-3 r\left(9^{\lambda-2} n\right) .
$$

Thus, if $n \equiv 11(\bmod 24)$,

$$
\begin{aligned}
r\left(9^{\lambda} n\right) & =4 r\left(9^{\lambda-1} n\right)-3 r\left(9^{\lambda-2} n\right) \\
& =4 \cdot 3^{\lambda-1} r(n)-3 \cdot 3^{\lambda-2} r(n) \\
& =\left(4 \cdot 3^{\lambda-1}-3 \cdot 3^{\lambda-2}\right) r(n) \\
& =3^{\lambda} r(n),
\end{aligned}
$$

if $n \equiv 19(\bmod 24)$,

$$
\begin{aligned}
r\left(9^{\lambda} n\right) & =4 r\left(9^{\lambda-1} n\right)-3 r\left(9^{\lambda-2} n\right) \\
& =4\left(2 \cdot 3^{\lambda-1}-1\right) r(n)-3\left(2 \cdot 3^{\lambda-2}-1\right) r(n) \\
& =\left(4\left(2 \cdot 3^{\lambda-1}-1\right)-3\left(2 \cdot 3^{\lambda-2}-1\right)\right) r(n) \\
& =\left(2 \cdot 3^{\lambda}-1\right) r(n),
\end{aligned}
$$

while if $n \equiv 3$ or $51(\bmod 72)$,

$$
\begin{aligned}
r\left(9^{\lambda} n\right) & =4 r\left(9^{\lambda-1} n\right)-3 r\left(9^{\lambda-2} n\right) \\
& =4\left(\left(3^{\lambda}-1\right) / 2\right) r(n)-3\left(\left(3^{\lambda-1}-1\right) / 2\right) r(n) \\
& =\left(4\left(\left(3^{\lambda}-1\right) / 2\right)-3\left(\left(3^{\lambda-1}-1\right) / 2\right)\right) r(n) \\
& =\left(\left(3^{\lambda+1}-1\right) / 2\right) r(n),
\end{aligned}
$$

and our result is proved.

Appendix. In [3], a generalisation of the following identity was established:

$$
\begin{aligned}
\sum a^{s+t+u} q^{\left(s^{2}+t^{2}+u^{2}\right) / 2} & \\
= & \left(-a q^{1 / 2} ; q\right)_{\infty}^{3}\left(-a^{-1} q^{1 / 2} ; q\right)_{\infty}^{3}(q ; q)_{\infty}^{3} \\
= & c_{0}\left(-a^{3} q^{3 / 2} ; q^{3}\right)_{\infty}\left(-a^{-3} q^{3 / 2} ; q^{3}\right)_{\infty}\left(q^{3} ; q^{3}\right)_{\infty} \\
& +c_{1}\left\{a\left(-a^{3} q^{5 / 2} ; q^{3}\right)_{\infty}\left(-a^{-3} q^{1 / 2} ; q^{3}\right)_{\infty}\left(q^{3} ; q^{3}\right)_{\infty}\right. \\
& \left.+a^{-1}\left(-a^{3} q^{1 / 2} ; q^{3}\right)_{\infty}\left(-a^{-3} q^{5 / 2} ; q^{3}\right)_{\infty}\left(q^{3} ; q^{3}\right)_{\infty}\right\}
\end{aligned}
$$

where

$$
c_{0}=1+6 \sum_{n \geq 1}\left(\frac{q^{3 n-2}}{1-q^{3 n-2}}-\frac{q^{3 n-1}}{1-q^{3 n-1}}\right) \quad \text { and } \quad c_{1}=3 q^{1 / 2} \frac{\left(q^{3} ; q^{3}\right)_{\infty}^{3}}{(q ; q)_{\infty}} .
$$


Thus we have

$$
\begin{aligned}
\sum q^{s^{2}+s t+t^{2}} & =\sum q^{\left(s^{2}+t^{2}+(-s-t)^{2}\right) / 2}=\sum_{s+t+u=0} q^{\left(s^{2}+t^{2}+u^{2}\right) / 2} \\
& =\text { the constant term in } \sum a^{s+t+u} q^{\left(s^{2}+t^{2}+u^{2}\right) / 2} \\
& =c_{0} \\
& =1+6 \sum_{n \geq 1}\left(\frac{q^{3 n-2}}{1-q^{3 n-2}}-\frac{q^{3 n-1}}{1-q^{3 n-1}}\right)
\end{aligned}
$$

and

$$
\begin{aligned}
\sum q^{s^{2}+s t+t^{2}+s+t} & \\
& =\sum q^{\left(s^{2}+t^{2}+(-s-t-1)^{2}-1\right) / 2} \\
& =q^{-1 / 2} \sum_{s+t+u=-1} q^{\left(s^{2}+t^{2}+u^{2}\right) / 2} \\
& =q^{-1 / 2} \cdot \text { the coefficient of } a^{-1} \text { in } \sum a^{s+t+u} q^{\left(s^{2}+t^{2}+u^{2}\right) / 2} \\
& =q^{-1 / 2} c_{1}=3 \frac{\left(q^{3} ; q^{3}\right)_{\infty}^{3}}{(q ; q)_{\infty}}
\end{aligned}
$$

Addendum. We conjecture that for each odd prime $p$, each $\lambda \geq 1$, each $\mu \in\{0,1, \ldots, p-1\}$ and all $n \geq 0$,

- if $p \equiv 1(\bmod 8)$, then

$$
\begin{aligned}
& t\left(p^{2 \lambda+1} n+\left(3 p^{2 \lambda}-3\right) / 8+\mu p^{2 \lambda}\right) \\
& = \begin{cases}p^{\lambda} t(p n+\mu) & \text { if } 8 \mu+3 \text { is a q.r. modulo } p, \\
\frac{(p+1) p^{\lambda}-2}{p-1} t(p n+\mu) & \text { if } 8 \mu+3 \text { is a q.n.r. modulo } p,\end{cases} \\
& t\left(p^{2 \lambda+2} n+\left(3 p^{2 \lambda+1}-3\right) / 8+\mu p^{2 \lambda+1}\right) \\
& =\frac{p^{\lambda+1}-1}{p-1} t\left(p^{2} n+(3 p-3) / 8+\mu p\right) \quad \text { for } \mu \neq(3 p-3) / 8,
\end{aligned}
$$

- if $p \equiv 3(\bmod 8)$, then

$$
\begin{aligned}
& t\left(p^{2 \lambda+1} n+\left(3 p^{2 \lambda}-3\right) / 8+\mu p^{2 \lambda}\right) \\
& = \begin{cases}p^{\lambda} t(p n+\mu) & \text { if } 8 \mu+3 \text { is a q.n.r. modulo } p, \\
\frac{(p+1) p^{\lambda}-2}{p-1} t(p n+\mu) & \text { if } 8 \mu+3 \text { is a q.r. modulo } p,\end{cases} \\
& t\left(p^{2 \lambda+2} n+\left(p^{2 \lambda+1}-3\right) / 8+\mu p^{2 \lambda+1}\right) \\
& =\frac{p^{\lambda+1}-1}{p-1} t\left(p^{2} n+(p-3) / 8+\mu p\right) \quad \text { for } \mu \neq(3 p-1) / 8,
\end{aligned}
$$


- if $p \equiv 5(\bmod 8)$, then

$$
\begin{aligned}
& t\left(p^{2 \lambda+1} n+\left(3 p^{2 \lambda}-3\right) / 8+\mu p^{2 \lambda}\right) \\
& = \begin{cases}p^{\lambda} t(p n+\mu) & \text { if } 8 \mu+3 \text { is a q.r. modulo } p, \\
\frac{(p+1) p^{\lambda}-2}{p-1} t(p n+\mu) & \text { if } 8 \mu+3 \text { is a q.n.r. modulo } p,\end{cases} \\
& t\left(p^{2 \lambda+2} n+\left(7 p^{2 \lambda+1}-3\right) / 8+\mu p^{2 \lambda+1}\right) \\
& =\frac{p^{\lambda+1}-1}{p-1} t\left(p^{2} n+(7 p-3) / 8+\mu p\right) \quad \text { for } \mu \neq(3 p-7) / 8,
\end{aligned}
$$

- if $p \equiv 7(\bmod 8)$, then

$$
\begin{aligned}
& t\left(p^{2 \lambda+1} n+\left(3 p^{2 \lambda}-3\right) / 8+\mu p^{2 \lambda}\right) \\
& = \begin{cases}p^{\lambda} t(p n+\mu) & \text { if } 8 \mu+3 \text { is a q.n.r. modulo } p, \\
\frac{(p+1) p^{\lambda}-2}{p-1} t(p n+\mu) & \text { if } 8 \mu+3 \text { is a q.r. modulo } p,\end{cases} \\
& t\left(p^{2 \lambda+2} n+\left(5 p^{2 \lambda+1}-3\right) / 8+\mu p^{2 \lambda+1}\right) \\
& =\frac{p^{\lambda+1}-1}{p-1} t\left(p^{2} n+(5 p-3) / 8+\mu p\right) \quad \text { for } \mu \neq(3 p-5) / 8 .
\end{aligned}
$$

In terms of $r(n)$, these relations are:

- if $p \equiv 1(\bmod 8)$, then

$$
r\left(p^{2 \lambda} n\right)=\left\{\begin{array}{l}
p^{\lambda} r(n) \\
\text { for } n \equiv 8 \mu+3(\bmod 8 p) \text { if } 8 \mu+3 \text { is a q.r. modulo } p, \\
\frac{(p+1) p^{\lambda}-2}{p-1} r(n) \\
\quad \text { for } n \equiv 8 \mu+3(\bmod 8 p) \text { if } 8 \mu+3 \text { is a q.n.r. modulo } p, \\
\frac{p^{\lambda+1}-1}{p-1} r(n) \\
\text { for } n \equiv 3 p(\bmod 8 p), n \neq 3 p^{2}\left(\bmod 8 p^{2}\right),
\end{array}\right.
$$

- if $p \equiv 3(\bmod 8)$, then

$$
r\left(p^{2 \lambda} n\right)=\left\{\begin{array}{l}
p^{\lambda} r(n) \\
\text { for } n \equiv 8 \mu+3(\bmod 8 p) \text { if } 8 \mu+3 \text { is a q.n.r. modulo } p, \\
\frac{(p+1) p^{\lambda}-2}{p-1} r(n) \\
\quad \text { or } n \equiv 8 \mu+3(\bmod 8 p) \text { if } 8 \mu+3 \text { is a q.r. modulo } p, \\
\frac{p^{\lambda+1}-1}{p-1} r(n) \\
\text { for } n \equiv p(\bmod 8 p), n \neq 3 p^{2}\left(\bmod 8 p^{2}\right),
\end{array}\right.
$$


- if $p \equiv 5(\bmod 8)$, then

$$
r\left(p^{2 \lambda} n\right)=\left\{\begin{array}{l}
p^{\lambda} r(n) \\
\quad \text { for } n \equiv 8 \mu+3(\bmod 8 p) \text { if } 8 \mu+3 \text { is a q.r. modulo } p, \\
\frac{(p+1) p^{\lambda}-2}{p-1} r(n) \\
\text { for } n \equiv 8 \mu+3(\bmod 8 p) \text { if } 8 \mu+3 \text { is a q.n.r. modulo } p, \\
\frac{p^{\lambda+1}-1}{p-1} r(n) \\
\text { for } n \equiv 7 p(\bmod 8 p), n \neq 3 p^{2}\left(\bmod 8 p^{2}\right),
\end{array}\right.
$$

- if $p \equiv 7(\bmod 8)$, then

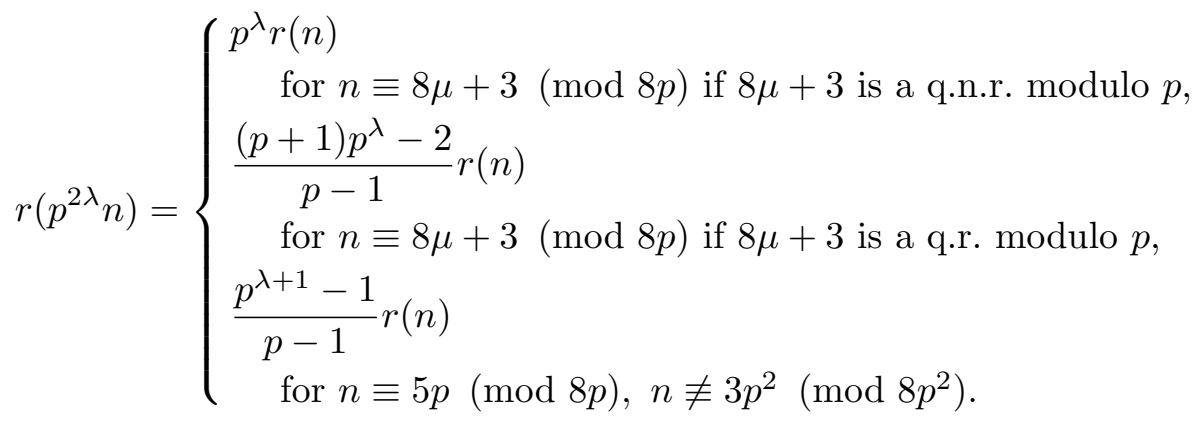

\section{References}

[1] G. E. Andrews, EYPHKA! num $=\Delta+\Delta+\Delta$, J. Number Theory 23 (1986), 285-293.

[2] C. F. Gauss, Werke, Bd. 10, Teubner, Leipzig, 1917, 497.

[3] M. Hirschhorn, F. Garvan and J. Borwein, Cubic analogues of the Jacobian theta function $\theta(z, q)$, Canad. J. Math. 45 (1993), 673-694.

School of Mathematics University of New South Wales Sydney 2052, New South Wales Australia

E-mail: m.hirschhorn@unsw.edu.au
Department of Science and Mathematics Cedarville College P.O. Box 601 Cedarville, Ohio 45314 U.S.A. E-mail: sellersj@cedarville.edu 